\title{
Backjumping is Exception Handling
}

\author{
ED ROBBINS and ANDY KING \\ University of Kent, Canterbury, CT2 7NF, UK \\ (e-mails: edd.robbins@gmail.com, a.m.king@kent.ac.uk) \\ JACOB M. HOWE \\ City, University of London, EC1V OHB, UK \\ (e-mail: j.m.howe@city.ac.uk)
}

submitted 15 September 2017; accepted 24 August 2020

\begin{abstract}
ISO Prolog provides catch and throw to realize the control flow of exception handling. This pearl demonstrates that catch and throw are inconspicuously amenable to the implementation of backjumping. In fact, they have precisely the semantics required: rewinding the search to a specific point and carrying of a preserved term to that point. The utility of these properties is demonstrated through an implementation of graph coloring with backjumping and a backjumping SAT solver that applies conflict-driven clause learning.
\end{abstract}

KEYWORDS: backjumping, exception handling, conflict-driven clause learning, SAT

\section{Introduction}

The ISO Prolog reference manual (Deransart et al. 1996) explains how catch and throw can pass control from one point of the program to another. The default behavior of catch(Goal, Catcher, RecoveryGoal) is to simply invoke Goal. However, if during execution, there is a call to throw (Ball) then control (and bindings) are unwound to the closest ancestor catch in the call stack which matches against the term Ball. Specifically, if the Catcher argument of the closest catch unifies with a copy of Ball, then the RecoveryGoal meta-call of that catch is invoked. Otherwise, control is unwound further until a matching catch is found. Since bindings are undone as the call stack is unwound, $\mathrm{Ball}$ might also be used to communicate information to RecoveryGoal, for example, to report the nature of a failure.

The power of this control flow construct is that it can transfer control to a specific point in the call stack using the Ball to target a specific catch. This is exactly what is required for backjumping. Backjumping (Stallman and Sussman 1977; Gaschnig 1979), in contrast to chronological backtracking, leaps across multiple levels in a search tree directly to the decision that triggered failure, rather than stepping through each decision, one by one. Backjumping has found application in truth maintenance systems (De Kleer 1986), logic programming (Bruynooghe 1980), constraint solving (Dechter 1990), and most recently in SAT to realize (Marques-Silva and Sakallah 1996) conflict-driven clause 
learning (CDCL). A CDCL solver requires not only search to be unwound to a specific decision (by backjumping), but also a term (a learnt clause) to be preserved and carried to that decision. Fortuitously, this facility is also provided by catch and throw.

The problem of adding control to the logic of a search algorithm sits at the very heart of logic programming (Kowalski 1979). However, how control is added, and the clarity of the control component, can be controversial. The problem of adding control is particularly acute when programming search problems like SAT, where the problem statement can be very simple, but the best algorithms analyze the decisions to focus search (as in CDCL). Moreover, backjumping is at odds with chronological backtracking, and as a consequence certain classes of algorithms that at first glance appear well suited to logic programming are, in fact, almost incompatible with the paradigm, at least in its purest form.

This pearl proposes catch and throw for programming backjumping, work that grew out of the (irritating) problem of how to clearly code CDCL in Prolog. This chimes with Bentley who coined the term programming pearl, and wrote, "Just as natural pearls grow from grains of sand that have irritated oysters, these programming pearls have grown from real problems that have irritated real programmers" (Bentley 1986). In contrast to a previous pearl (Bruynooghe 2004), which used a mutable database to orchestrate all aspects of intelligent backtracking, this paper breaks down the problem of implementing CDCL into its various components which are then matched against the language constructs of Prolog. The net result is clarity. To be precise, CDCL decomposes into three components: (1) rewinding search and bindings, (2) communication of a newly learned clause to its insertion point in the search tree, and (3) retaining learned clauses (across backtracking and backjumping). This paper argues that catch and throw provide (1) and (2), whereas (3) is naturally provided by a mutable database that might be implemented with a dynamic predicate, blackboard (De Bosschere and Jacquet 1993), or non-backtrackable global variables (Wielemaker et al. 2012). Applications are not limited to SAT, or even SMT (Robbins et al. 2015); to demonstrate versatility, the approach is first illustrated on the classic problem of graph coloring, providing a template for backjumping with catch and throw, then, second it is applied for SAT with learning.

Catch and throw have been advocated for programming backjumping before as part of a comp.lang.prolog discussion (Baljeu 2005), but the authors are not aware of any studies which actually demonstrate the viability of the idea. Deploying catch and throw in backjumping is unconventional since exception handling is intended to support exceptional behavior, whereas in backjumping these constructs are used for the intended control flow, which is in turn exceptional in the context of Prolog's execution model. The discussion of Baljeu (2005) is centered on the use of these non-logical ISO language features in Prolog programming.

The rest of this paper is structured as two case studies on backjumping, the first on graph coloring and the second on SAT. The graph coloring study has been chosen as a minimal example of depth-first search with backjumping, and the code provides a template for other examples. Section 2 explains how catch and throw can be used to realize backjumping for a graph coloring problem, where the edge constraints are realized as tests which check the color assigned to each vertex as it becomes bound. Section 3 moves onto SAT, building on the template provided by the graph coloring study to 

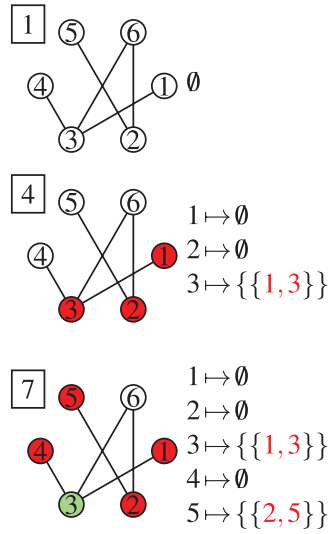

$1 \mapsto \emptyset$

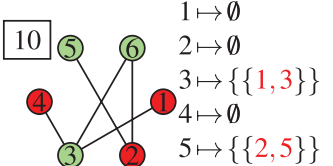
$6 \mapsto\{\{2,6\},\{3,6\}\}$
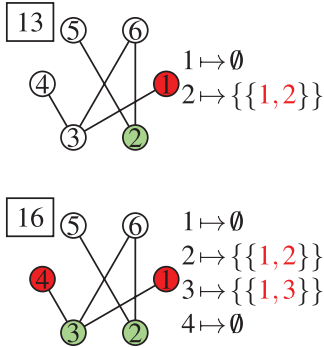
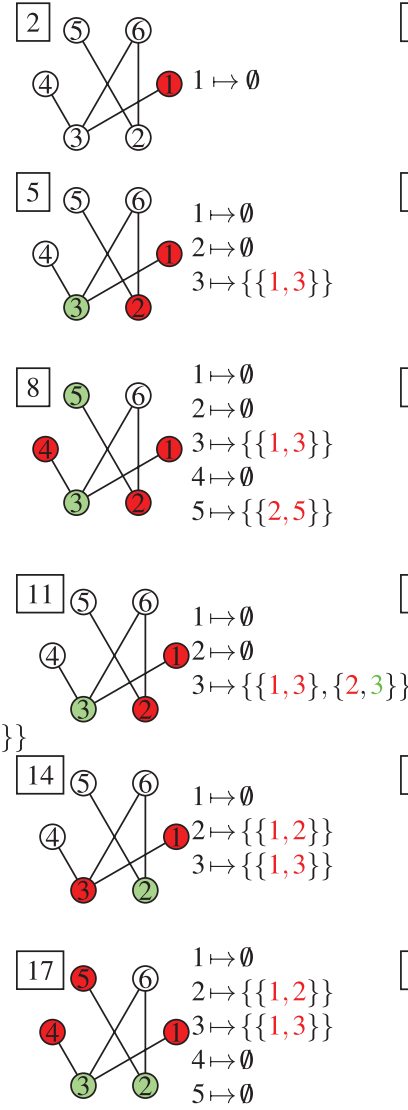
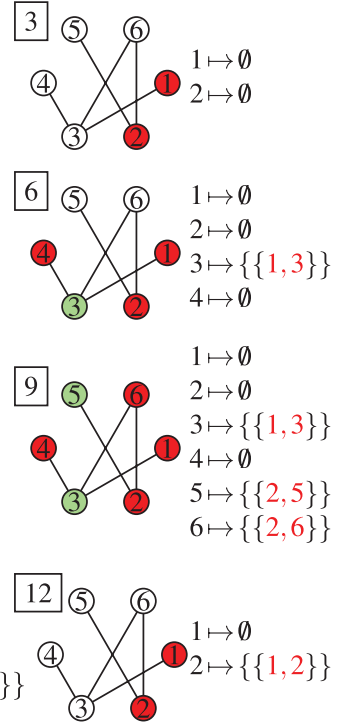
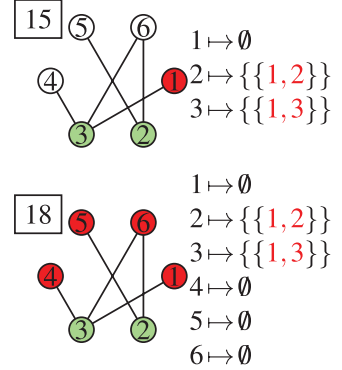

Fig. 1. Graph coloring with backjumping.

illustrate how catch and throw can be deployed to communicate (learnt) information back to the catch, necessary when guiding search using CDCL. Section 4 presents the concluding discussion.

\section{Graph coloring}

The first of the two worked examples in this paper considers graph coloring, adding backjumping to depth-first search. This example has been chosen as a minimal illustrating example, with the code in Figure 3 providing a template for other search problems, including the motivating example of SAT solving with CDCL.

Figure 1 illustrates depth-first search with backjumping for a coloring problem, where the objective is to assign red or green to each of the six vertices of the graph so that vertices which share an edge are colored differently. The example uses just two colors for simplicity. The vertices of the graph are ordered, as indicated by the numbering. Coloring commences at vertex 1, red is tried before green at each vertex, and white indicates the absence of a color assignment. Each partially colored graph is augmented with a map which associates each vertex with a (possibly empty) set of conflicts. A conflict is a 
set of vertices with a color assignment that cannot be extended to satisfy all the edge constraints.

The conflict map is initially empty and is extended as each vertex is colored and contracted on backtracking and backjumping. The first conflict occurs in diagram 4 of Figure 1 between vertices 1 and 3 and is recorded in the conflict map for vertex 3 ; the conflict is always logged on the most recently assigned vertex. For expositional purposes, 1 and 3 are colored red indicating the partial color assignment when the conflict is detected.

Green has yet to be tried for vertex 3; hence, backtracking is applied to undo the assignment at vertex 3 and reassign it green (diagram 5 ). The conflict $\{1,3\}$ is retained: the assignment to vertex 1 is still red but vertex 3 is now green. Thus, both vertices of the conflict, with the possible exception of the last, preserve their initial colors, which is a general pattern.

The next conflict arises at vertex 5 (diagram 7 ), causing the conflict $\{2,5\}$ to be recorded. Not all colors have been considered at vertex 5 so again backtracking undoes the assignment to vertex 5 and it is reassigned green. Next, vertex 6 is colored red, which conflicts with vertex 2 (diagram 9). Again, not all colors have been tried at vertex 6 , thus the vertex is reassigned to green, which then conflicts with vertex 3 (diagram 10). Notice that the first vertices (in the vertex ordering) of $\{2,5\},\{2,6\}$, and $\{3,6\}$ retain their initial color.

\subsection{Conflict analysis for graph coloring}

Now that all colors have been tried at vertex 6 , backjumping is deployed after a form of conflict analysis which infers the target of the backjump. The conflicts for vertex 6 are $\{2,6\}$ and $\{3,6\}$, indicating that the conflicts at vertex 6 involve the assignments of vertices 2,3 , and 6 , but not vertices 4 and 5 . Moreover, one conflict occurs when vertices 2 and 6 are both red, and the other occurs when vertices 3 and 6 are both green. Hence, $\{2,3\}$ is also a conflict, where 2 is red and 3 is green, since this partial assignment is incompatible with the edge constraints, irrespective of the color assigned to vertex 6 . Therefore, a solution cannot be found without either reassigning the color at vertex 2 , or vertex 3 , or both. The conflict set of vertex 3 is augmented with $\{2,3\}$ to give $\{\{1,3\},\{2,3\}\}$ (diagram 11). Since vertex 3 was assigned more recently than vertex 2 , it is selected as the target of the backjump and search resumes at vertex 3 .

It should be noted that although the vertices of each conflict are colored in Figure 1, it is not necessary to introduce additional color assignments, one per conflict, to record these colors. To see this, observe how the colors of 2 and 3 in $\{2,3\}$ align with the current color assignment because the 2 of $\{2,6\}$ and the 3 of $\{3,6\}$ also match with the current color assignment. Thus, the first vertices of both $\{1,3\}$ and $\{2,3\}$ match the current color assignment. Hence, the colors of the vertices of a conflict, with the possible exception of the last, match those of the current color assignment, a property which holds inductively.

Vertex 3 has already been assigned to both red and green, so again a form of conflict analysis is applied to infer that the partial assignment on the vertices 1 and 2 cannot be extended to a complete solution, whatever the color of vertex 3 . Therefore, $\{1,2\}$ is also a conflict, which is associated with vertex 2 . Note how the colors of $\{1,2\}$, which are both red, match the current color assignment. This conflict cannot be remedied without reassigning either vertex 1 or vertex 2 or both. The higher of the two, vertex 2 , is thus 
taken as the target of the backjump, which is then colored green (diagram 13). Conflicts on vertex 3 result in reassignment of that vertex to green (diagram 15), before the search proceeds to find a complete satisfying color assignment (diagram 18).

Finally, observe that the map data structure can be simplified by replacing each set of conflicts with a single set which is the union of all its conflicts (Bruynooghe 2004). Thus, the map for diagrams $9,10,11$, and 12 , are replaced by:

$$
\begin{aligned}
& 1 \mapsto \emptyset \quad 1 \mapsto \emptyset \\
& 2 \mapsto \emptyset \quad 2 \mapsto \emptyset \\
& 3 \mapsto\{1,3\} \quad 3 \mapsto\{1,3\} \\
& 4 \mapsto \emptyset \quad 4 \mapsto \emptyset \\
& 5 \mapsto\{2,5\} \quad 5 \mapsto\{2,5\} \\
& 6 \mapsto\{2,6\} \quad 6 \mapsto\{2,3,6\} \\
& \begin{array}{ll}
1 \mapsto \emptyset \\
2 \mapsto \emptyset
\end{array} \quad 1 \mapsto \emptyset \\
& 3 \mapsto\{1,2,3\} \quad 2 \mapsto\{1,2\}
\end{aligned}
$$

Observe how entry $3 \mapsto\{1,2,3\}$ of the third map given immediately above can be found by unioning the vertex sets for $3 \mapsto\{1,3\}$ and $6 \mapsto\{2,3,6\}$ of its predecessor and then eliminating vertex 6 , which is the vertex whose coloring induced the conflict analysis. In fact, not all the map needs to be accessed simultaneously: only the set of vertices for the highest identifier. This allows the map to be organized as a stack of lists, where only the topmost list of vertices on the stack is modified at any one time. This gives a straightforward data structure for implementing conflict analysis.

Notice that it is possible that an assignment leads to several conflicts. Here, the standard approach is taken - one conflict is selected to inform the backjump, and it is possible that one of the other conflicts is then encountered as search continues. An alternative approach would be to record all conflicts and backjump to the shallowest point in the search tree to guarantee that none of these would be encountered again. In the Prolog implementation considered in the next section, if there are several conflicts, the scheduler will determine which conflict is first encountered and used to make the backjump. The correctness of backjumping is addressed by Kondrak and van Beek (1997).

\subsection{Backjumping in Prolog}

Coloring can be realized by adopting a test-and-generate model in which checks suspend on their variables until they become sufficiently instantiated to apply the test. These checks then coroutine with a generator phase which binds the variables, one by one, that represent the colors of the vertices. SICStus code is provided to achieve this, where Figure 2 sets up the checks which define the coloring problem and Figure 3 applies labeling, with backjumping, to search for a satisfying assignment.

Setting up the checks for coloring. The predicate colour(Vars, Values, Cs) solves a coloring problem, where Vars is a list that specifies the color which is assigned to each vertex, Values defines the range of colors that can be assigned at each vertex, and Cs is a list of disequalities specifying the edge constraints. For the problem instance in Figure 1, the initial call would have

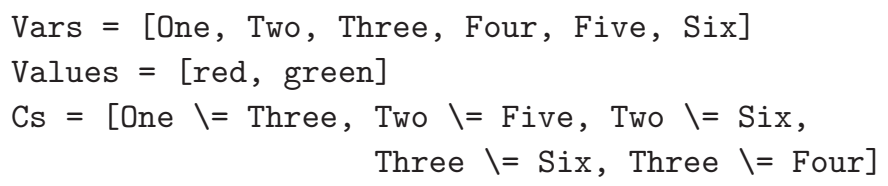




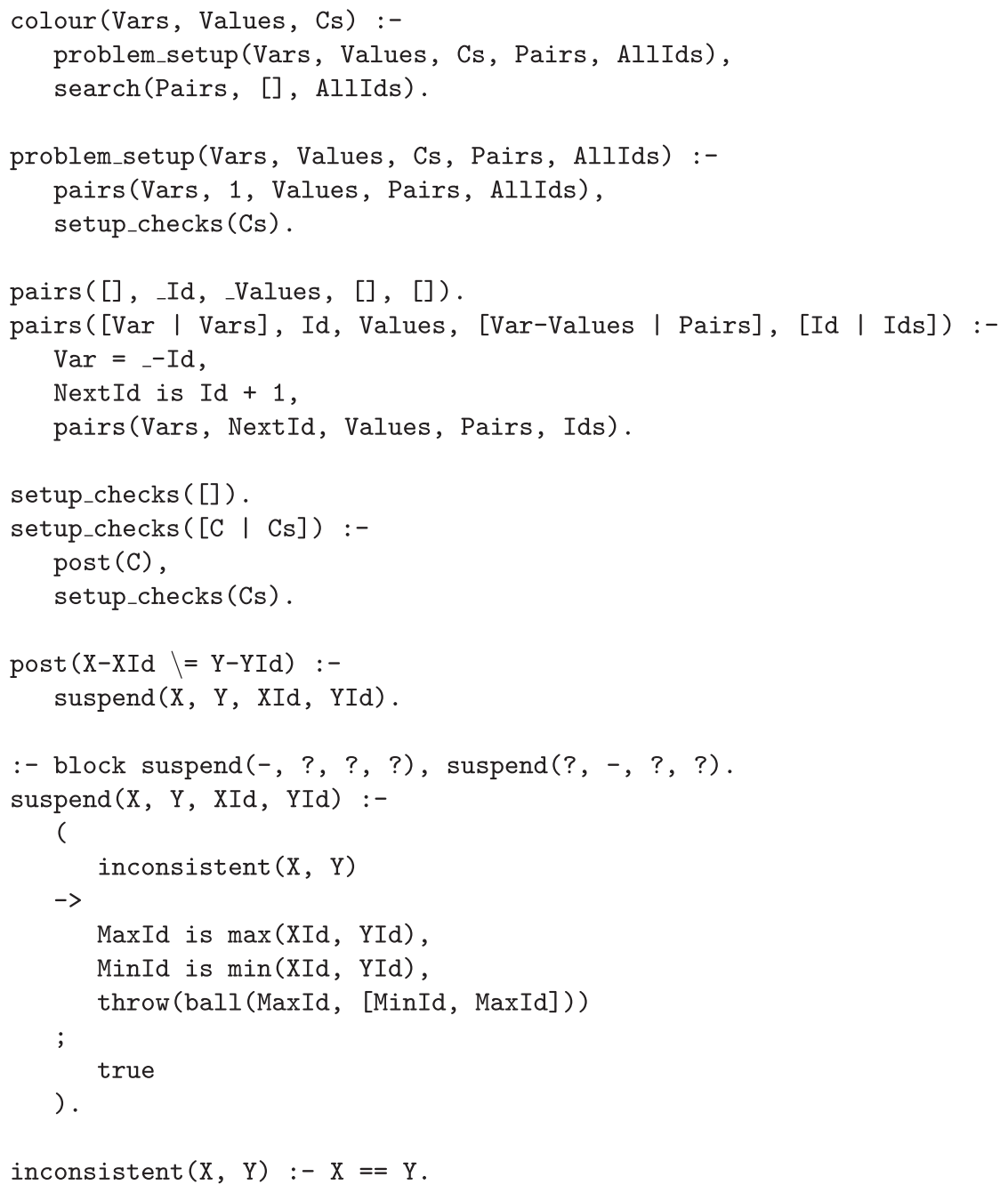

Fig. 2. Setting up the checks for graph coloring.

The initial call first invokes problem_setup(Vars, Values, Cs, Pairs, AllIds) that is responsible for setting up Vars, Pairs, and AllIds and has as its second goal, search(Pairs, [], AllIds) for controlling the search. In setting up the problem, the predicate pairs(Vars, 1, Values, Pairs, AllIds) instantiates each element of the Pairs list to a term Var-Values where Var is itself instantiated to a pair Value-Id such that Value is drawn from the list Values and Id is a (ground) identifier; AllIds is instantiated to a list of all identifiers. The identifiers are numeric, indicating the position of Var-Values within Pairs, which tallies with the order in which variables are (later) assigned. The predicate setup_checks(Cs) posts each check given in a list of disequalities Cs. Each goal post (X-XId $\backslash=\mathrm{Y}-\mathrm{YId}$ ) invokes suspend(X, Y, XId, YId) whose block declaration specifies that the suspend goal should not be called until both $\mathrm{X}$ and $\mathrm{Y}$ are instantiated. If inconsistency is detected on instantiation, then the term ball (MaxId, CIds) is thrown where MaxId is the largest identifier of the set CIds formed from XId and YId which identify the variables involved in the conflict. Note that corou- 


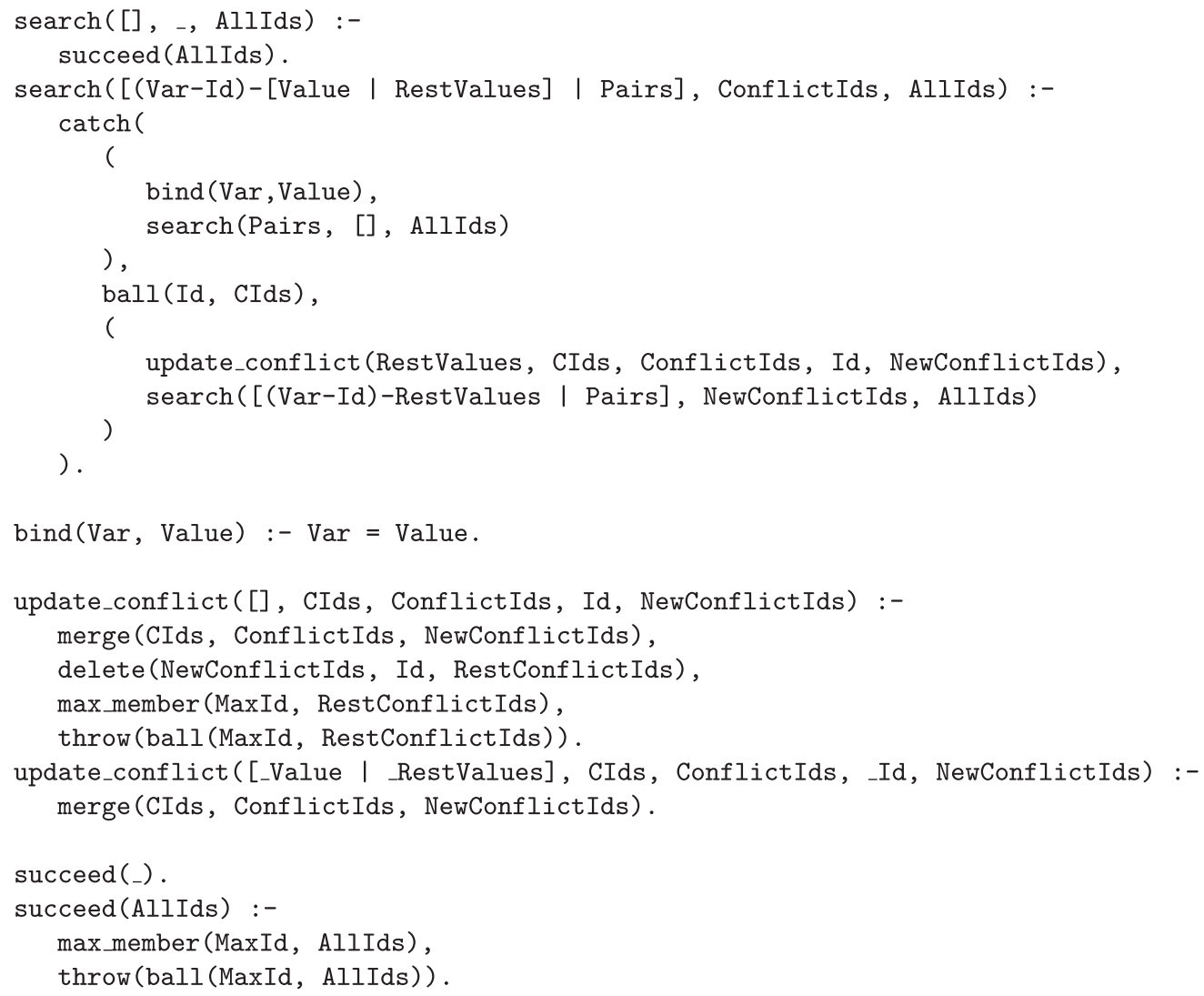

Fig. 3. Backjumping search for graph coloring.

tining is described here using SICStus's block declarations (Carlsson and Mildner 2012). Although coroutining is not part of the ISO standard, other mainstream Prolog systems provide similar control constructs for delaying goals, such as when or freeze.

Backjumping for coloring. The predicate search(Pairs, ConflictIds, and AllIds) assigns the variables of Pairs to satisfy the constraints, failing if there is no solution. The ConflictIds argument maintains a conflict set for the variable of the first pair of Pairs.

Consider first the second clause of search which is responsible for orchestrating backjumping. Each meta-call catch(Goal, Catcher, and RecoveryGoal) has Goal that is concerned with assigning one variable, identified by Id, to the color Value. Binding Var to Value might wake up blocked calls to suspend, which will lead to consistency checks. If binding Var to Value does not lead to inconsistency, then search proceeds to search for an assignment to the remaining Pairs. If inconsistency is discovered, ball (MaxId, CIds) is thrown by a check in suspend and the call stack is unwound to the first enclosing catch for which Catcher unifies with ball(MaxId, CIds). The Catcher term of each meta-call is ball ( Id, CIds) where Id is ground and CIds is a variable, thus any catch which intercepts ball (MaxId, CIds) must possess an identifier Id which matches MaxId. In fact, this realizes backtracking; if a call to bind assigns Var (with identifier Id), wakes 
suspend in Figure 2 and inconsistency is discovered, then both variables are instantiated; hence, MaxId must be Id and the catch directly enclosing the call to bind handles the exception. This then allows the conflict information in CIds to be passed back to the point where search resumes. An earlier ancestor will only intercept a ball thrown in update_conflict which is realizing backjumping. Note that bind(Var, Value) can unblock several suspend goals. Yet when the first goal resumes it will throw its ball, undoing the binding, so that the other suspend goals become blocked again.

RecoveryGoal has two calls, the first to update_conflict maintains conflict information for backjumping and the second continues search. If there are further colors to be tried, the second clause of update_conflict merges the conflict information for the current failure with that for previous failures, without duplication. The call to search will then assign the next color from RestValues. If there are no further colors to be tried, that is RestValues is empty, backjumping should occur. The first clause of update_conflict enables this. Conflict information is merged, the current assignment identifier is removed from the conflict list, and then the highest identifier remaining is the backjump level; hence, this and the conflict information are thrown as ball (MaxId, RestConflictIds). This achieves backjumping by unwinding the call stack to where the variable with identifier MaxId is bound, while communicating the new conflict RestConflictIds to that part of the search. At this point, either a color remains to be assigned or backjumping is again applied, and so search continues. Observe that undoing and then reassigning a variable to another color, which is the essence of backtracking, is realized entirely using catch and throw.

If the first clause of search is matched, then all variables will have been assigned a color. This clause of search invokes the goal succeed(AllIds), which will immediately succeed thereby returning the solution to the coloring problem. If another answer is requested, then a throw is used to reactivate search, the MaxId selected from AllIds which is list of all the variable identifiers. This results in search backtracking into the nonconflicting solution. The call to succeed(AllIds) can be omitted if it is sufficient to compute a single answer. It should be noted that all control is provided by catch and throw: it is not necessary to resort to a mutable database to maintain the conflicts and direct search (Bruynooghe 2004).

Search as presented in Figures 2 and 3 provides a template for implementing backjumping. Predicate search wraps catch (Goal, Catcher, RecoveryGoal) where the role of Goal is to bind variables to values, but if conflicts arise they are described by the term ball (Id, CIds) and caught by Catcher, and then the role of RecoveryGoal is to update conflict information and either continue search, or backjump, as appropriate.

\section{SAT}

Figure 4 lists the code for a Prolog SAT solver, adapted from Howe and King (2010; 2012) that uses watched literals to realize unit propagation. Given a propositional formula $f$ in CNF over a set of variables $X$, and a partial (truth) function $\theta: X \rightarrow\{$ true, false $\}$, unit propagation examines each clause of $f$ to deduce another partial function $\theta^{\prime}: X \rightarrow$ $\{$ true, false $\}$ that extends $\theta$ and that, if $\theta$ can be extended to satisfy $f$, necessarily holds. For example, suppose $X=\{x, y, z, u, v, w\}, f=(\neg x \vee z \vee \neg y) \wedge(\neg z \vee \neg u) \wedge(u \vee w \vee$ $\neg v) \wedge(\neg w \vee v)$ and $\theta=\{x \mapsto$ true, $y \mapsto$ true $\}$. In this instance, the clause $(\neg x \vee z \vee \neg y)$ 


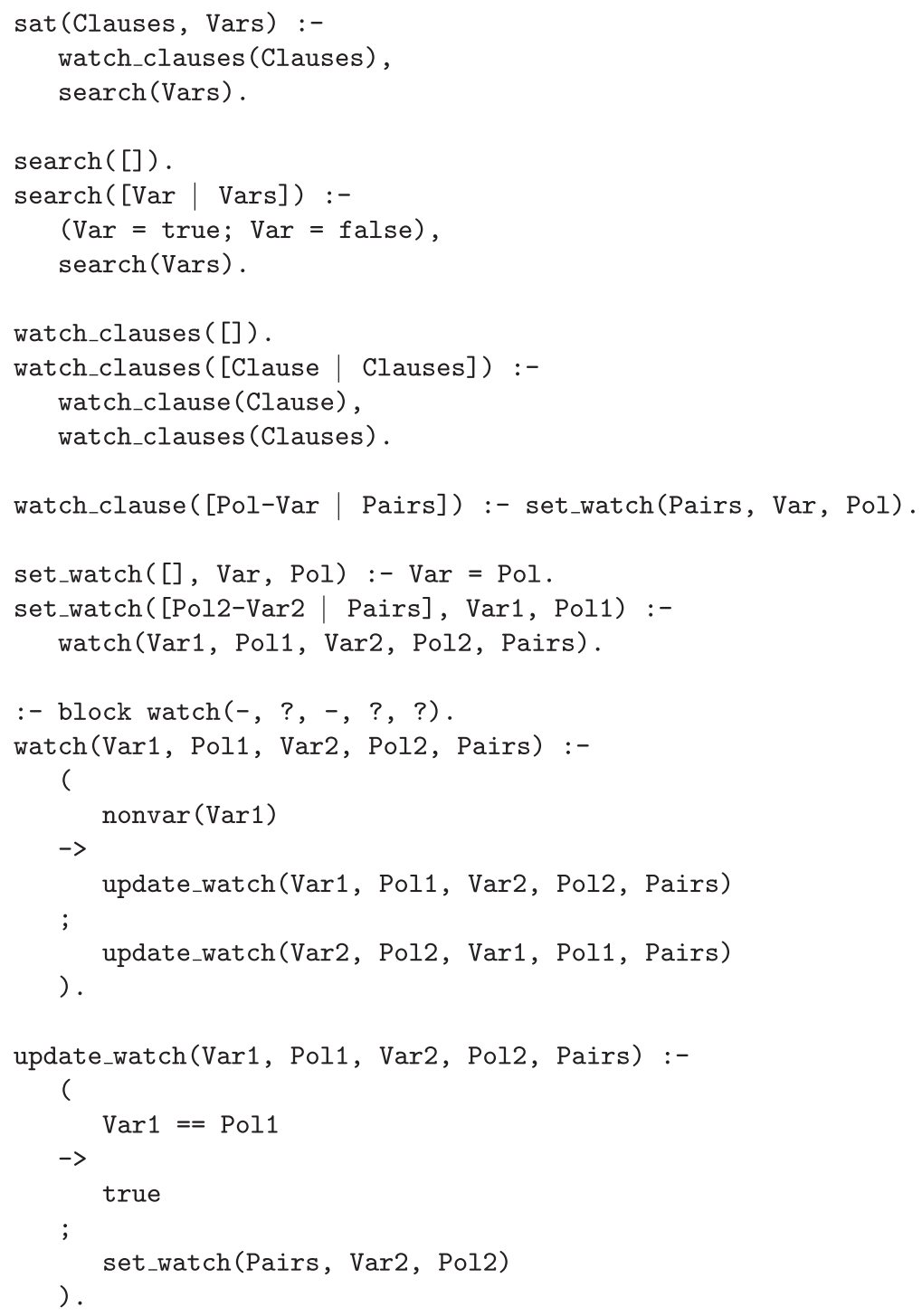

Fig. 4. A (vanilla) SAT solver using watched literals Howe and King (2010; 2012).

is unit, because it has only one unbound variable, $z$. Therefore, it can be deduced that, given $\theta$, for the clause to be satisfied $z \mapsto$ true. Moreover, for $(\neg z \vee \neg u)$ to be satisfied, it follows that $u \mapsto$ false. The satisfaction of the remaining two clauses depends on two unknowns, $v$ and $w$; hence, no further information can be deduced from them. Therefore, $\theta^{\prime}=\theta \cup\{z \mapsto$ true, $u \mapsto$ false $\}$.

Searching for a satisfying assignment of $f$ proceeds as follows: starting from an empty truth function $\theta=\emptyset$, unit propagation is applied to $\theta$ until either no further propagation is possible or a contradiction is established. In the first case, if all clauses are satisfied then $f$ is satisfied, else an unassigned variable occurring in $f$, for instance $x$, is selected and the assignment $x \mapsto$ true is added to $\theta$. In the second case, search backtracks to a 
previous assignment, $y \mapsto$ true say, then adds $y \mapsto$ false to $\theta$ and continues with unit propagation.

The watched literals technique is founded on the simple observation that a particular clause is unit if it does not contain two unassigned variables (Moskewicz et al. 2001). Therefore, for each clause of a problem, two unassigned variables are watched; propagation may occur once either is assigned. It is not enough to watch just one variable because this is, in general, not sufficient for detecting if a clause becomes unit: the watch might be on the other, unassigned variable. The SAT solver in Figure 4 takes a problem in CNF, specified as a list of clauses, and a list of variables. Each clause is itself a list of pairs Pol-Var, where Var is a propositional variable, and Pol indicates whether the variable has positive or negative polarity by being either true or false, respectively. For the introductory example above, the initial call to sat/2 would have

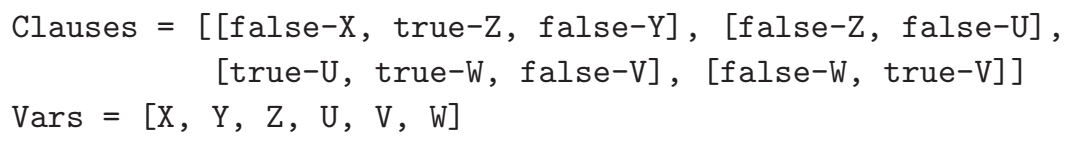

For each clause, the watch_clause predicate invokes set_watch that, in turn, selects the first two variables in the clause to be watched. In the above, for the first clause. this leads to the invocation of watch (X, false, Z, true, [false-Y]); with neither X nor Z instantiated, this goal suspends via a delay declaration (specified using block in SICStus syntax). When a variable is instantiated, watch resumes and executes update_watch. If the instantiated variable matches its polarity, the clause is satisfied, and update_watch exits successfully, otherwise another variable is selected for watching. For the clause being considered, if $X$ is bound to true, then set_watch ([false-Y], Z, true) will be called which in turn will lead to the suspended watch (Z, true, Y, false, []). If only one unbound variable remains, set_watch realizes unit propagation and assigns that variable so that the clause is satisfied. So if $Y$ is bound to true, after waking watch, set_watch instantiates $\mathrm{Z}$ to be true by unit propagation; further, the second clause leads to U being instantiated to false. If there are no unassigned variables, or assigned and satisfying variables, then the clause is unsatisfiable and the set_watch goal will fail, and search will backtrack. If the example being considered is further extended by binding $\mathrm{V}$ to true, then the third clause will infer by unit propagation that $\mathrm{W}$ is instantiated to true. The fourth clause, which was suspended as watch(W, false, V, true, [] ) will wake and lead to the call set_watch([], true, false), which fails. The search is realized using the search predicate to assign variables to true or false. The search for a satisfying assignment then proceeds as previously described, simply through Prolog backtracking.

\subsection{Backjumping in a Prolog SAT solver}

The goal of this paper is to augment the Prolog SAT solver in Figure 4 with CDCL. This is achieved by following the backjumping template from the previous section, where alongside the backjump, learnt clauses are added to the problem description. This section describes how backjumping for SAT is built, including the subtleties arising from accommodating clause learning. Figures 5, 6, 7, 8, and 10 extend the SAT solver of Figure 4 with infrastructure for backjumping and conflict analysis. 


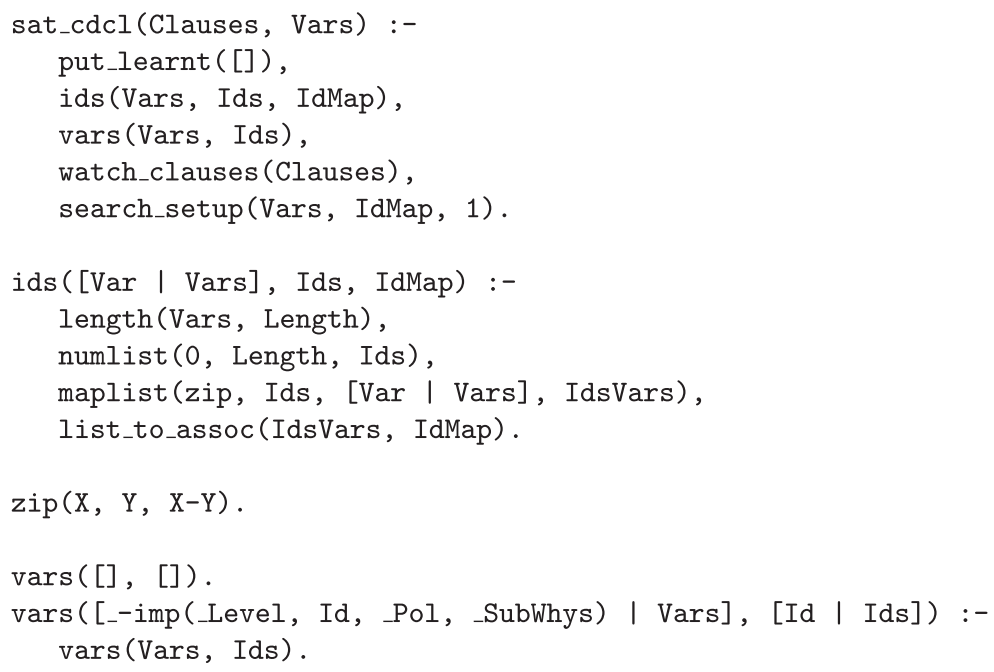

Fig. 5. Initial call and setup.

Variables and implication graphs. In Figure 5, the initial call to the CDCL solver is given, along with the setup of the variables. An identifier map, IdMap, which associates a ground identifier with the variable it represents, is created during setup to later reconstruct clauses during learning. This mapping is constructed up-front prior to invoking watch_clauses. An implication graph (Marques-Silva et al. 2009) for a variable Var is conceptually a DAG in which each node is represented as a term imp(Level, Id, Value, Whys) where Level records the decision level at which Var was assigned; Id is a (ground) identifier that is unique to the variable (used solely for deriving a ground representation of a learnt clause); Value is the truth value bound to the variable; and Whys is itself a list of implication graphs, interpreted as subtrees. Each propositional variable of the vanilla solver is replaced with a compound term Var-Why that pairs the variable Var with an implication graph Why that explains its instantiation. At the setup stage, the Why term of each pair Var-Why is unified with imp(_Level, Id, _Value,_Whys) where Id is the identifier for Var that ensures that each implication graph always carries its identifier.

Recall that in coloring, the decision level at which a variable is instantiated matches the position of the variable in the list Vars which also corresponds to its identifier. A consequence of unit propagation, however, is that the instantiation order does not necessarily follow the ordering of Vars; hence, the decision level does not necessarily match the identifier. Therefore, Level and Id are separately recorded in the imp structure. Furthermore, a learning solver reasons about the order in which variables are instantiated. Since, even at the same decision level, the instantiation of one variable can trigger the instantiation of another, the first element of imp is actually a pair Level-SubLevel where the integer SubLevel records the order in which a variable is instantiated within a given Level.

Setting up propagators for SAT. Figure 6 presents an enhanced version of watch_clause that supports conflict analysis. Unit propagation is extended to record the reason why a propositional variable is bound to a particular truth value. Since the Var argument of 


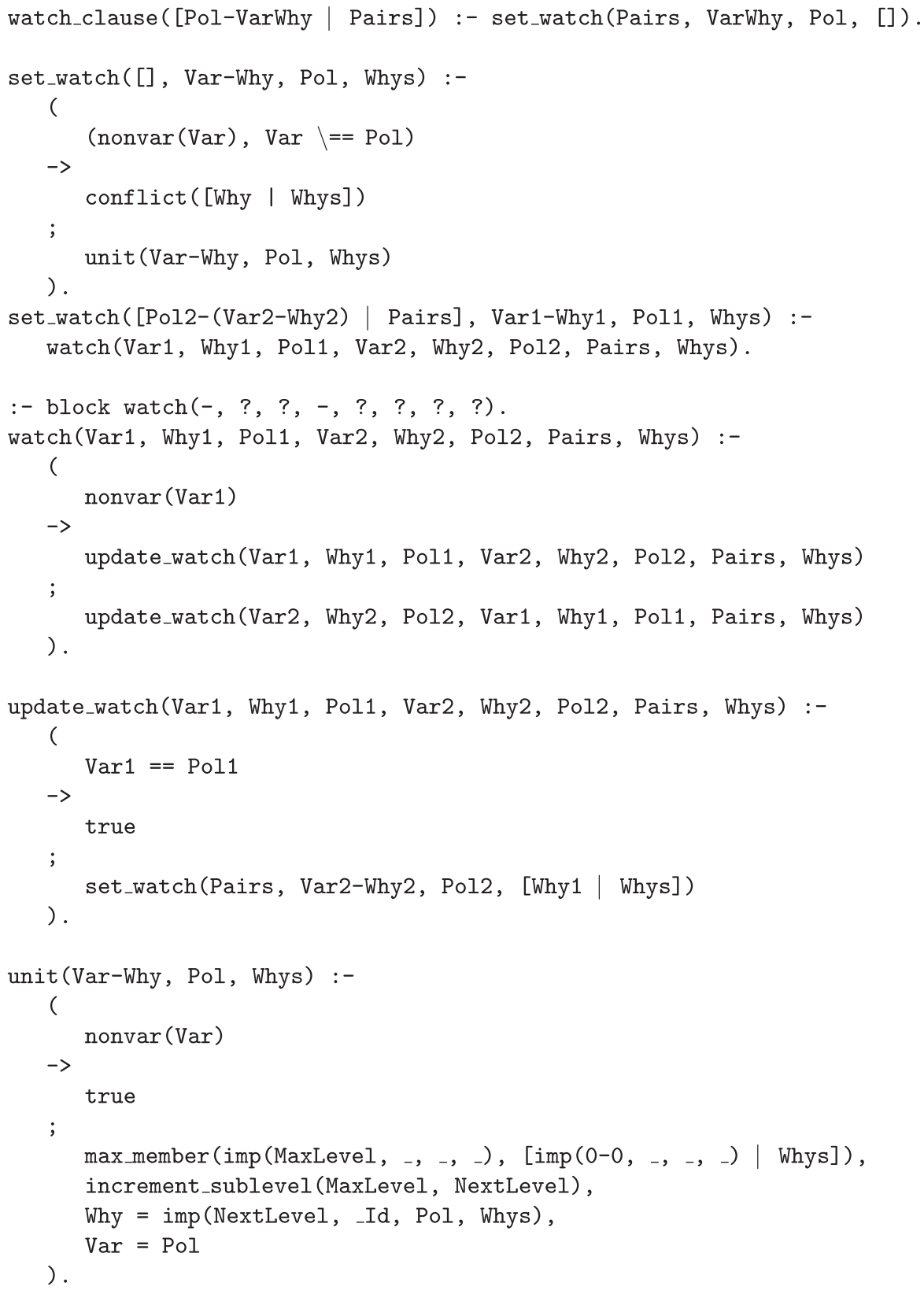

Fig. 6. Setting up the propagators for SAT.

watch_clause is replaced by a pair Var-Why, the two Var1 and Var2 arguments of watch are accompanied with two additional arguments Why1 and Why2. Then, the goal conflict uses the Why for Var and a list of implication graphs, Whys, for the other variables of the clause to diagnose the cause of the conflict, which is summarized as a clause. The goal ultimately terminates by throwing a ball which includes the learnt clause or triggers 
failure when an inconsistency is found with the clauses posted during setup. Notice how Whys is accumulated as each clause is traversed in update_watch.

The unit(Var-Why, Pol, Whys) goal calculates the decision level for a variable as part of the construction of its implication graph why. This predicate is only invoked from set_watch when Var is either uninstantiated or bound to Pol. The latter case is vacuous, but the former case of unit binds Var to the truth value Pol and creates why which records the reason for the binding. The max_member predicate harvests the maximum of the levels of implication graphs Whys of the other variables collected as the clause is traversed. The additional $\operatorname{imp}\left(0_{-} 0_{2}, \ldots, \ldots\right)$ term ensures that MaxLevel is well defined even when inconsistency is detected prior to any assignment by search. The predicate increment_sublevel merely increments the sublevel. The unification Var = Pol follows the bind to Why to ensure that every instantiated propositional variable is associated with a complete implication graph.

Search with backjumping for SAT. Figure 7 gives code which realizes backjumping search for SAT. The main search predicate is search (VarWhys, IdMap, Level) where, like before, VarWhys is a list of pairs, but here each pair is a variable conjoined with an implication graph which explains its binding. Search is controlled by overlaying backjumping with learning, in which the reason for a conflict is summarized by a conjunction of propositional literals whose negation gives a clause that is implied by the SAT instance. The clause (often referred to as a blocking clause) is then added to the problem to steer search away from the conflict. The map IdMap associates a ground identifier with the variable it represents, needed to reconstruct a (non-ground) clause from a conflict. Level is the decision level which a variable adopts if it is assigned by search rather than propagation. The first decision level is taken to be 1 (though, as discussed later, decision level 0 can also occur).

Consider first the second clause of search which is responsible for backjumping, learning, and labeling. Each catch meta-call is concerned with assigning one variable if it is unassigned. The predicate bind realizes labeling and instantiates Var to a truth value, while recording the value and level in a (leaf) node of an implication graph. Note that bind is never backtracked into, and assignment to false is not explicitly made. Since this code is realizing CDCL, failure of a binding will lead to a blocking clause being learnt and added to the problem description which will guide search away from the assignment that has failed, directing search to the false branch. If a conflict is discovered during search, the set_watch predicate in Figure 6 will call conflict(Whys) in Figure 7 and a ball is created with BackjumpLevel instantiated to the decision level of the backjump. The throw will thus only match the unique Catcher term ball (Level, Clause) for which Level $=$ BackjumpLevel. The ground BackjumpLevel and Clause terms are formed by the predicate analyse_conflict(Whys, BackjumpLevel, Clause) using a conflicting list of implication graphs Whys discovered when watching a clause (see Section 3.2 and Figure 10). On recovery, the bindings made by search and propagation are unwound back to the backjump level as required (though the bindings on the ball are retained). The RecoveryGoal then uses update_conflict in Figure 8 to add Clause to a database of learnt clauses (the learning algorithm explained in Section 3.2) which grows as search proceeds. The ground Clause is translated into its non-ground representation at the stage of the RecoveryGoal where the decision level reverts to that of BackjumpLevel: 


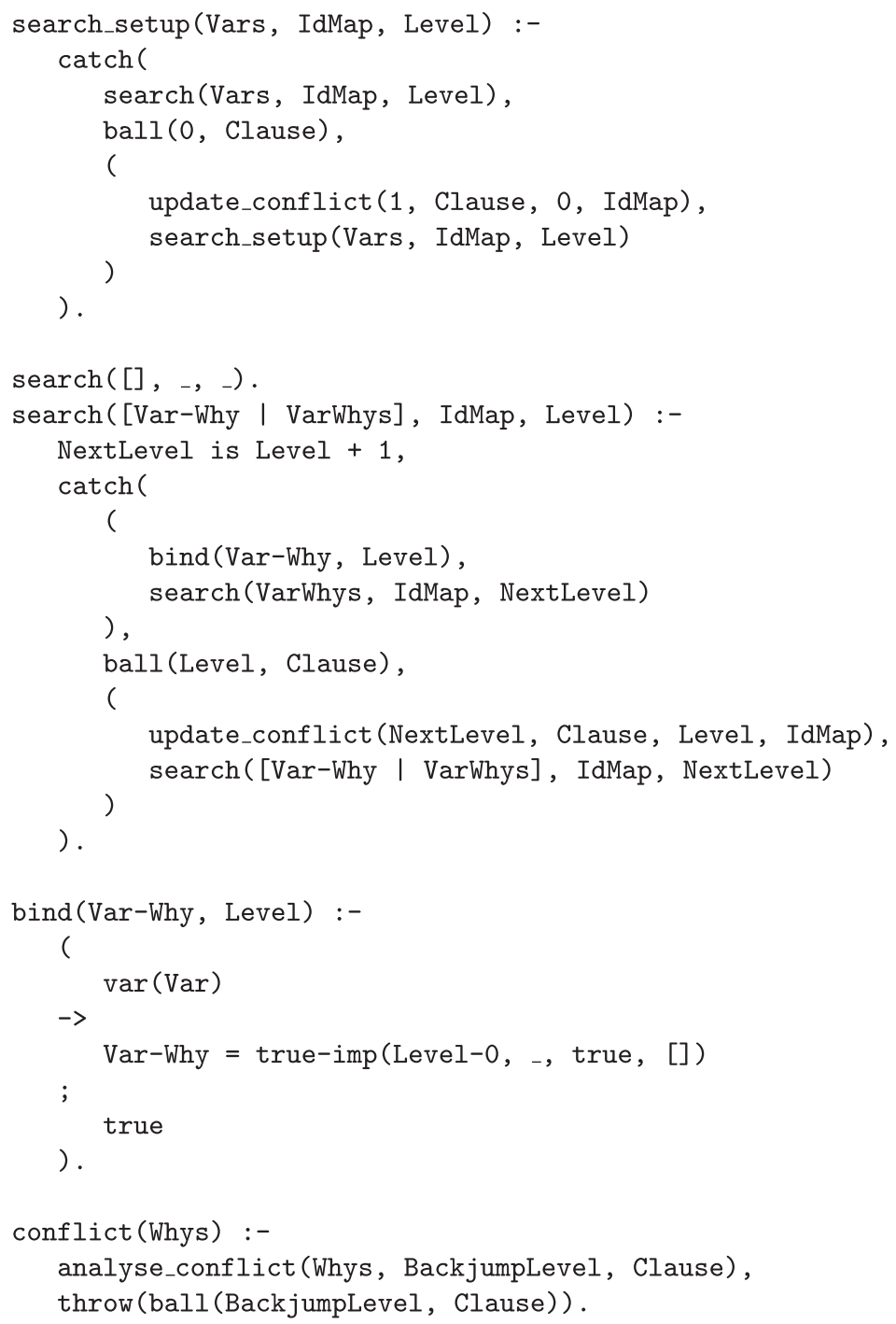

Fig. 7. Backjumping search for SAT.

the variables of the clause can have bindings at later decision levels which do not hold at the BackjumpLevel. Other elements of the database of learnt clause may also need to be restored in a similar way.

The predicates get_learnt(Learnt) and put_learnt(Learnt) are merely wrappers to built-ins that read and write a list of (learnt) clauses to a non-backtrackable database: they can be realized with bb_get/bb_put as illustrated in Figure 8, or nb_getval/nb_setval (Wielemaker et al. 2012), or using the assert/retract family of built-ins.

When considering graph coloring in Section 2, colors are always explicitly assigned in a search phase, and a conflict can only occur during search, after a color is assigned. However, SAT is different: a variable might be assigned a value before search at the initial setup. The decision level of such an assignment is taken to be 0 . Conflicts which arise 


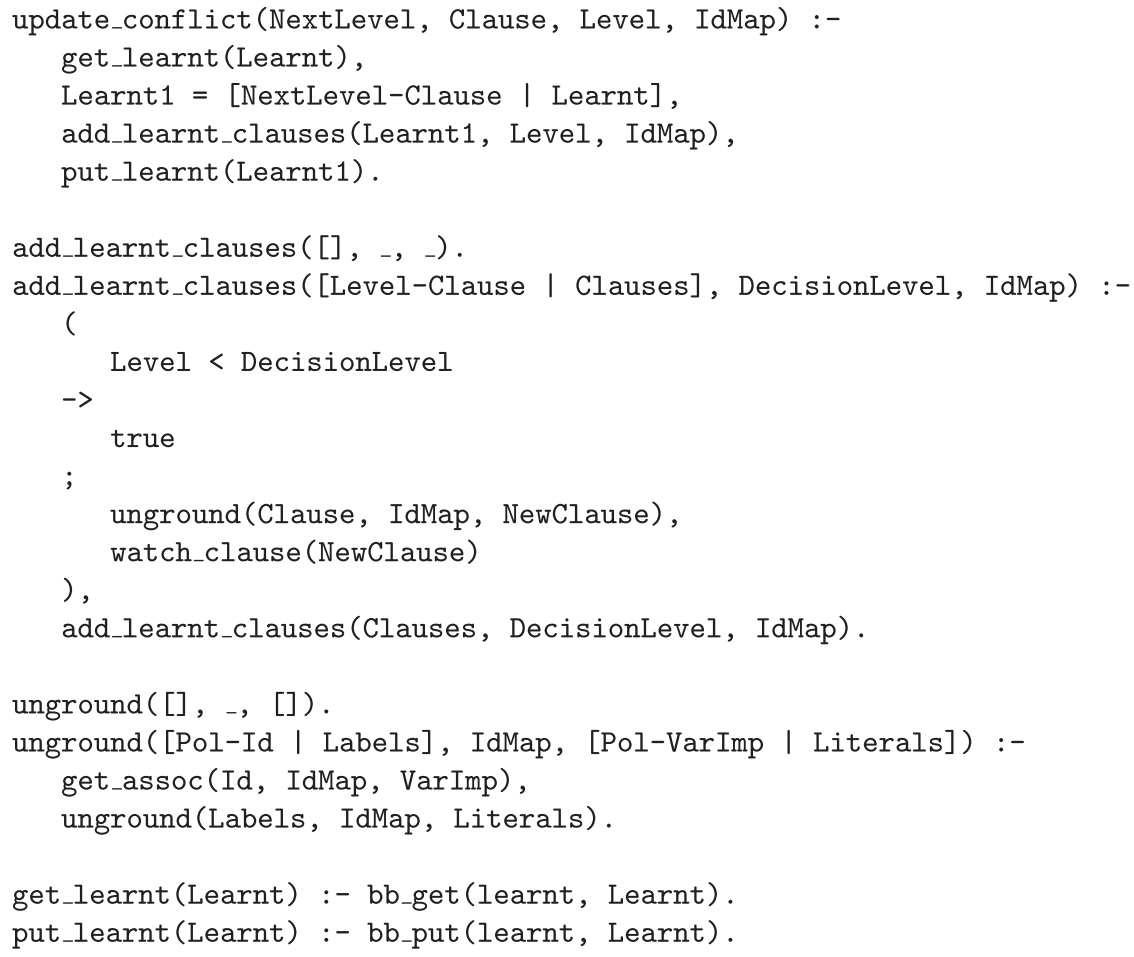

Fig. 8. Conflict analysis.

at this level are handled by the singleton case in analyse_conflict, which immediately fails without reaching a throw, since if the problem specification is unsatisfiable then no further search is necessary. Furthermore, with assignment at decision level 0 possible, conflict analysis might determine that level 0 is the appropriate decision level to jump to. This is prior to the call to search, hence the addition of search_setup to handle these backjumps; this predicate mirrors search, but without the call to bind. In addition, it is possible that the backjump caught by ball ( $0, \mathrm{Clause})$ describes a unit Clause and this itself leads to assignments at decision level 0.

\subsection{Conflict analysis for SAT}

When a conflict is encountered, the implication graph leading to it is examined to learn a clause, which is added to the formula to steer search away from the conflict. To illustrate, consider the formula $f=\left(\neg x_{1} \vee x_{8} \vee \neg x_{2}\right) \wedge\left(\neg x_{1} \vee \neg x_{3}\right) \wedge\left(x_{2} \vee x_{3} \vee x_{4}\right) \wedge\left(\neg x_{4} \vee \neg x_{5}\right) \wedge\left(x_{5} \vee\right.$ $\left.x_{6}\right) \wedge\left(x_{7} \vee \neg x_{4} \vee \neg x_{6}\right)$ and the partial assignment $\theta=\left\{x_{7} \mapsto\right.$ false, $x_{8} \mapsto$ false $\}$ where the variables $x_{7}$ and $x_{8}$ are assigned at decision levels 1 and 2 , respectively, by search. Observe that no further bindings are inferred by unit propagation. However, if search subsequently adds $x_{1} \mapsto$ true at decision level 3 , then a series of unit propagations ensue that ultimately lead to a conflict, owing to unsatisfiability of the clause $\left(x_{7} \vee \neg x_{4} \vee \neg x_{6}\right)$.

Unique Implication Points. Figure 9 illustrates an implication graph rooted at a special conflict node, $\kappa$, that details how the conflict follows from binding $x_{4}, x_{6}$, and $x_{7}$. Each 


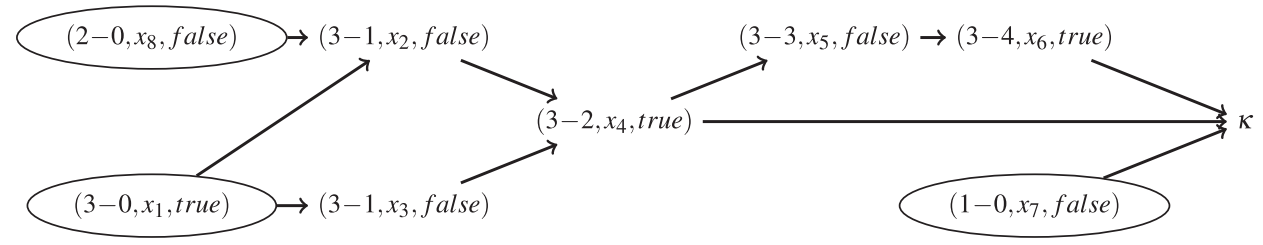

Fig. 9. Implication graph from $f$ and the partial assignment $\theta=\left\{x_{7} \mapsto\right.$ false, $x_{8} \mapsto$ false,$x_{1} \mapsto$ true $\}$.

triple in the figure gives the level and sublevel, the variable assigned, and its truth value. The implication graph can be inspected to learn a clause. The leaves of the implication graph, which are circled in Figure 9, are the three bindings $x_{7} \mapsto$ false, $x_{8} \mapsto$ false, and $x_{1} \mapsto$ true, that together with clauses of $f$, prohibit the satisfiability of $\left(x_{7} \vee \neg x_{4} \vee \neg x_{6}\right)$. This combination of bindings can be avoided by adding $\left(x_{7} \vee x_{8} \vee \neg x_{1}\right)$ to $f$ which is therefore a candidate learnt clause. However, another choice is possible, notably one with fewer literals. Observe that any path that starts with the binding of $x_{1}$ at decision level 3 , the current decision level, and ends at $\kappa$ passes through the intermediate node where $x_{4}$ is assigned. The single binding $x_{4} \mapsto$ true therefore summarizes the net effect of the two bindings $x_{1} \mapsto$ true and $x_{8} \mapsto$ false. Thus, an alternative learnt clause is $\left(\neg x_{4} \vee x_{7}\right)$. In general, any node between the current (most recent) decision variable and the conflict $\kappa$ that strictly dominates (Cooper et al. 2006) $\kappa$ can be used to construct a learnt clause. Such nodes are termed Unique Implication Points (UIPs). The UIP nearest $\kappa$ is the first UIP (the node where $x_{4}$ is assigned). The last UIP is furthest from $\kappa$ and is where the current decision variable is bound (the node where $x_{1}$ is assigned).

Figure 10 presents code for creating a learnt clause based on the last UIP. The predicate construct_clause performs a depth-first traversal of the implication graph, starting from $\kappa$, and identifies decision variables by their empty implication graphs (SubWhys). The constructed clause is made up of literals where variables are identified by the Id ground term, and polarities are the negation of those that caused the conflict.

The construct_clause predicate also gathers the decision levels at which literals in the new clause were assigned. These levels are used to find the backjump level, which is chosen to be such that the learnt clause becomes unit, directing search away from the conflict. For the last UIP learnt clause $x_{7} \vee x_{8} \vee \neg x_{1}$ of Figure 9, construct_clause will derive the clause [false-x1, true-x8, true-x7] with decision levels [3, 2, 1] . Search should resume by backjumping to level 2. Therefore, the call to conflict (Whys) leads to throw (ball (2, [false-x1, true-x8, true-x7])). More generally, the backjump level is the largest level strictly less than the maximum (which is actually the current decision level); search would not immediately benefit from the new clause if resumed at an earlier decision level. Continuing at the backjump level ensures that the learnt clause becomes unit almost immediately.

The act of backjumping removes bindings, in particular those induced by recently added clauses. However, these clauses are retained in the non-backtrackable database using a ground representation. Figure 8 lists the code for reinstating learnt clauses after backjumping using the predicate add_learnt_clauses. Learnt clauses are saved together with the level at which they were learned. Upon backjumping, the calls to watch_clause for clauses learnt at the backjump decision level or later are lost, and these calls need to 


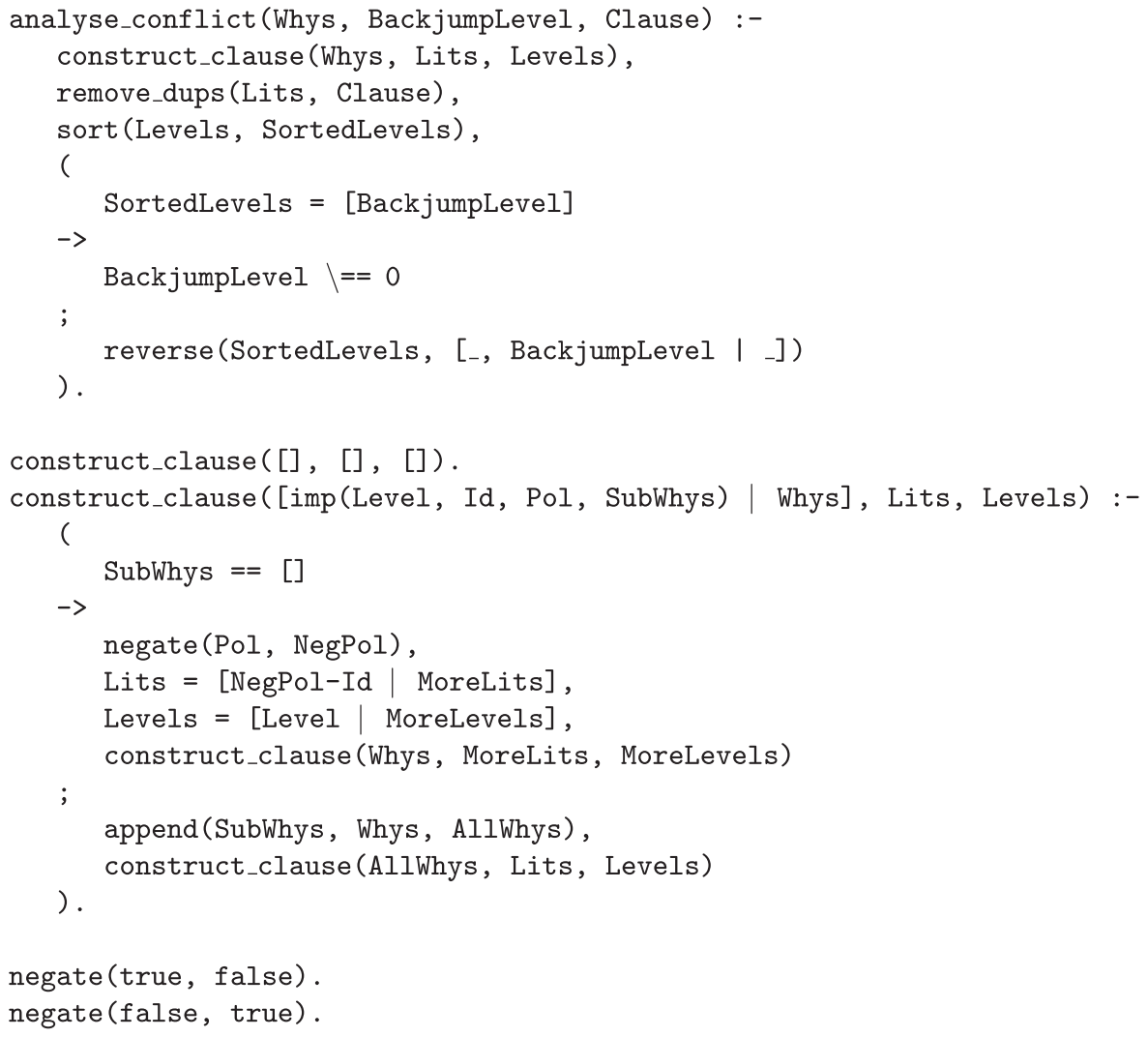

Fig. 10. Inferring and instantiating learnt clauses.

be made again. This necessitates rebuilding clauses after backjumping from the ground representation of the database. The unground predicate achieves this using association list IdMap to map a ground identifier to its propositional variable. This list is built during problem setup and is passed through search as shown in Figure 7.

Reinstatement of learned clauses can be performed selectively to realize $k$-learning. In $k$-learning (Marques-Silva and Sakallah 1996), learned clauses are only added to the constraint store permanently if they have less than $k$ variables. It decreases the cost of learning by lessening pressure on memory and reducing the number of updates to the store of clauses. Clauses with fewer variables have most influence, but learning will have little impact if $k$ is set too low. The presented approach can be also modified to allow first UIP clause learning. This typically produces smaller clauses more tightly focused on the cause of the conflict (Marques-Silva et al. 2009). First UIP can be realized in Prolog by running a frontier over the implication graph, starting at $\kappa$, repeatedly expanding the node with the highest level and sublevel. The first UIP is found when the frontier reduces to contain a single node at the highest level (possibly augmented with nodes of lower level). First UIP can thus be found in a single pass over the conflict graph without introducing additional data structures. 
Table 1. Performance of learning versus no learning

\begin{tabular}{lrrrrrrrr}
\hline \hline & \multicolumn{2}{c}{ No learning } & & \multicolumn{4}{c}{8 -learning } \\
\cline { 2 - 3 } Benchmark & Time & Assign & & Time & Assign & Throws & Jumps \\
\hline CBS_k3_n100_m435_b90_127 & 2987 & 179,427 & & 2206 & 56,329 & 24,650 & 31,667 \\
CBS_k3_n100_m435_b90_139 & 3204 & 193,247 & & 874 & 26,827 & 10,163 & 16,647 \\
flat175-17 & 22,791 & 748,377 & & 10,225 & 107,066 & 43,546 & 63,477 \\
flat175-28 & 15,754 & 471,521 & & 15,132 & 154,985 & 70,895 & 84,059 \\
flat200-20 & 16,345 & 519,868 & & 3252 & 39,674 & 16,107 & 23,524 \\
flat200-39 & 30,000 & & & 13,326 & 125,053 & 54,516 & 70,502 \\
uf100-0126 & 4097 & 248,581 & & 1984 & 53,320 & 23,009 & 30,293 \\
uf100-015 & 3499 & 210,330 & & 857 & 24,676 & 10,101 & 14,559 \\
uuf100-0119 & 9651 & 634,568 & & 3644 & 95,553 & 40,073 & 55,497 \\
uuf100-0120 & 5731 & 350,866 & & 2578 & 67,868 & 28,378 & 39,498 \\
\hline \hline
\end{tabular}

Backjumping is designed to accelerate search which begs the question of whether backjumping, when implemented with catch and throw, can ever improve on the default search mode of Prolog. Table 1 presents timings for a biased sample of classic SAT benchmarks; biased because they were chosen to be nontrivial in that the execution time exceeds $2 \mathrm{~s}$ for the vanilla solver. Times are in milliseconds, and benchmarking was carried out with SICStus 4.5.1 on a 2.5-GHz Macbook Pro with 16GB RAM. The first two benchmarks are random 3-SAT instances with controlled backbone size, the next four originate from flat graph coloring problems, and the final four include two unsatisfiable and two satisfiable random 3-SAT instances. The table gives data for the vanilla solver with no learning, and for the backjumping solver given in this paper, augmented with first UIP CDCL and using $k$-learning with $k=8$. For both solvers, the execution time in milliseconds is given (time), alongside the number of variable assignments made (assign). In addition, for the backjumping solver, the number of throws made by the solver (throws), and the number of assignments jumped over (jumps) are given. The balls thrown in backjumping are the size of the analyzed conflict which is typically less than 20 literals for these benchmarks, though this is problem-dependent. The timings suggest that catch and throw are not only a useful code structuring device but can enable significant improvement in performance when used to realize backjumping. As is conventional in SAT solving, the timings are for finding a single solution. To enumerate all solutions, the template from graph coloring using the succeed predicate can be adapted to add a blocking clause for the solution and reactivating search reusing the learnt clauses. Code for the work presented in this paper is available at https://www.cs.kent.ac.uk/ amk/ backjump.zip, including some variations not discussed here, such as pruning the set of learnt clauses by removing those which are entailed by a newly learnt clause.

\section{Concluding Discussion}

The idea of using catch and throw to realize backjumping dates back at least to a discussion (Baljeu 2005) on comp.lang.prolog but the authors are not aware of any programming examples that serve to illustrate the technique. The message of this discussion is exemplified with two case studies which demonstrate that catch and throw are more 
versatile than one would expect, providing exactly what is required for programming backjumping. In fact, random solver restarts (Howe and King 2012) can also be accommodated by adding an outer catch meta-call which intercepts restart exceptions thrown when the number of backjumps exceeds a threshold.

Intelligent backtracking (Bruynooghe 2004) can be combined with learning by restarting the search from scratch and then fast-forwarding to a particular decision point (Howe and King 2012). However, catch and throw provide a more general solution, not requiring search to be completely restarted, and there seems no reason why this strategy cannot extend to SMT solving (Robbins et al. 2015) by another instantiation of the template. It has been recently shown (Drabent 2018) that the vanilla SAT solver of Figure 4 can be understood as a logic program with added control; however, reasoning about the correctness of learnt clauses is more challenging still, a research problem that is not exclusive to logic programming.

Realizing backjumping with an ISO feature is undoubtedly attractive, and since ISO encourages the use of catch and throw, one might hope that increasingly efficient implementations will emerge over time. Even though SWI-Prolog does not comply with the ISO specification (since it does not copy the Ball before unifying it with Catcher), the two case studies are fully portable because the Goal and Catcher goals do not share variables.

\section{Supplementary material}

To view supplementary material for this article, please visit https://doi.org/10.1017/ S1471068420000435.

\section{Acknowledgments}

The authors thank the editor and anonymous referees for their thought-provoking comments. This is a better paper for these suggestions. This work was supported, in part, by EPSRC grants EP/K031929/1 and EP/N020243/1.

\section{References}

BAlJeu, A. 2005. Using Exceptions to Implement Backjumping? comp.lang.prolog newsgroup discussion. URL: http://coding.derkeiler.com/Archive/Prolog/comp.lang. prolog/2005-09/msg00048.html.

Bentley, J. 1986. Programming Pearls. Addison-Wesley: New Jersey.

Bruynooghe, M. 1980. Analysis of dependencies to improve the behaviour of logic programs. In International Conference on Automated Deduction. Lecture Notes in Artificial Intelligence, vol. 87. Springer, 293-305.

Bruynooghe, M. 2004. Enhancing a search algorithm to perform intelligent backtracking. Theory Practice of Logic Programming 4, 3, 371-380.

Carlsson, M. and Mildner, P. 2012. SiCStus Prolog - the first 25 years. Theory and Practice of Logic Programming 12, 1-2, 35-66.

Cooper, K. D., Harvey, T. J. And Kennendy, K. 2006. A Simple, Fast Dominance Algorithm. Technical Report TR-06-33870, Rice University, Computer Science. URL: http://www.cs. rice.edu/ keith/EMBED/dom.pdf.

De Bosschere, K. And Jacquet, J. 1993. Multi-Prolog: Definition, operational semantics and implementation. In International Conference on Logic Programming. MIT Press, 299-313. 
De Kleer, J. 1986. An Assumption-based TMS. Artificial Intelligence 28, 2, 127-162.

Dechter, R. 1990. Enhancement schemes for constraint processing: Backjumping, learning, and cutset decomposition. Artificial Intelligence 41, 273-312.

Deransart, P., Ed-Dbali, A. And Cervoni, L. 1996. Prolog: The Standard. Springer.

Drabent, W. 2018. Logic + Control: On program construction and verification. Theory Practice of Logic Programming 18, 1, 1-29.

Gaschnig, J. 1979. Performance Measurement and Analysis of Certain Search Algorithms. Ph.D. thesis, Carnegie Mellon University, Department of Computer Science, Pittsburgh, Pennsylvania 15213. CMU-CS-79-124.

Howe, J. M. And King, A. 2010. A pearl on SAT solving in Prolog. In Functional and Logic Programming. Lecture Notes in Computer Science, vol. 6009. Springer, 165-174.

Howe, J. M. And KIng, A. 2012. A pearl on SAT and SMT solving in Prolog. Theoretical Computer Science 435, 43-55.

Kondrak, G. AND van BeEK, P. 1997. A theoretical evaluation of selected backtracking algorithms. Artifical Intelligence 89, 365-387.

Kowalski, R. A. 1979. Algorithm = Logic + Control. Communication of the ACM 22, 7, 424-436.

Marques-Silva, J. P., Lynce, I. And Malik, S. 2009. Conflict-driven clause learning SAT solvers. In Handbook of Satisfiability, A. Biere, M. Heule, H. Van Maaren and T. Walsh, Eds. IOS Press, 131-153.

Marques-Silva, J. P. And Sakallah, K. A. 1996. GRASP - A new search algorithm for satisfiability. In Computer Aided Design. IEEE, 220-227.

Moskewicz, M., Madigan, C., Zhao, Y., Zhang, L. and Malik, S. 2001. Chaff: Engineering an efficient SAT solver. In Design Automation Conference. ACM Press, 530-535.

Robbins, E., Howe, J. M. And King, A. 2015. Theory propagation and reification. Science of Computer Programming 111, 1, 3-22.

Stallman, R. And Sussman, G. J. 1977. Forward reasoning and dependency-directed backtracking in a system for computer-aided circuit analysis. Artificial Intelligence 9, 135-196.

Wielemaker, J., Schrijvers, T., Triska, M. and Lager, T. 2012. SWI-Prolog. Theory and Practice of Logic Programming 12, 1-2, 67-96. 